\title{
Globales Bewusstsein und religiöse Identität: Positionen evangelischer Schülerinnen und Schülern in vier Kontinenten
}

\begin{abstract}
Zusammenfassung
Dieser Beitrag erörtert die Frage, wie Jugendliche globale Zusammenhänge wahrnehmen und welche Rolle religiöse Überzeugungen dabei spielen. Hierfür werden Statements von Schülerinnen und Schülern evangelischer Schulen aus vier Kontinenten analysiert und hinsichtlich deren Überzeugungen bezüglich gesellschaftlicher Fragen, Schule und Bildung, Religion und Kirche interpretiert. Darin zeigen sich zahlreiche Werte und Visionen, die teilweise religiös begründet werden; es werden aber auch regionale Unterschiede zwischen den Kontinenten erkennbar. In den pädagogischen Schlussfolgerungen wird eine Förderung der Sensibilisierung junger Menschen für globale Herausforderungen empfohlen.
\end{abstract}

Schlüsselworte: Wahrnehmung von Globalität, religiöse Identität, religiöse Bildung

\begin{abstract}
This article discusses the question of how young people perceive global connections and the role of religious beliefs in this context. Therefore, statements by students from Protestant schools on four continents are analysed and interpreted in terms of their convictions regarding social issues, school and education, religion and church. This reveals numerous values and visions, some of which are based on religious convictions, but also reveals regional differences between the continents. The educational conclusions recommend promoting young people's awareness of global challenges.
\end{abstract}

Keywords: GlobalAwareness, Religious Identit, Religious Education

\section{Einleitung}

Bei der Erörterung eines Themas wie dem in der vorliegenden Ausgabe dieser Zeitschrift, nämlich „Religiöse Bildung in einer globalisierten Welt", wird oftmals der Ausgangspunkt bei den Inhalten und den Lernzielen der Lehrplanung oder bei der akademischen Diskussion genommen. Was Schülerinnen und Schüler selbst zu diesem Thema denken, welche Kenntnisse,
Wertvorstellungen oder Urteile sie mitbringen, ist schwieriger zu erheben, aber von erheblicher Bedeutung für Bildungsziele und Lernprozesse. Wie nehmen Jugendliche globale Bezüge und Horizonte wahr? Wo sehen sie Verbindungen zwischen ihrem Alltag und weltweiten Zusammenhängen? Was bedeuten ihnen Bildung und Lernen in diesem Kontext? Welche Werte sind ihnen wichtig und welche Zukunftsvisionen haben sie? Welche Rolle spielen religiöse Überzeugungen dabei? Diesen Fragen soll in diesem Beitrag nachgegangen werden, indem Statements von Schülerinnen und Schülern an Schulen in evangelischer Trägerschaft in Afrika, Asien, Europa und Amerika ausgewertet werden, die im Rahmen eines Projektes zum Reformationsjubiläum entstanden. Im ersten Abschnitt werden der Hintergrund des Projekts, ein weltweiter Aufruf zur Einsendung von Statements sowie die Datengrundlage und Auswertungsmethodik skizziert. Daraufhin werden einige Ergebnisse im Überblick vorgestellt und im dritten Teil drei Sachbereiche ausführlicher analysiert:

- die Aspekte der Globalisierung

- die Rolle von Bildung

- die Bedeutung religiöser Werte und Überzeugungen.

Nach einer Zusammenfassung im vierten Abschnitt werden im letzten Teil einige Schlussfolgerungen für die pädagogische Praxis gezogen.

\section{Ein weltweiter Aufruf - Materialgrundlage und Auswertungsmethodik}

Das Material für den vorliegenden Beitrag beruht auf einer Aktion, die im Jahr 2016 im Kontext des weltweiten Netzwerks evangelischer Schulen (GPENreformation ${ }^{1}$ ) durchgeführt wurde. Schülerinnen und Schüler waren aufgerufen, unter dem Motto „Protest für die Zukunft“ ihre Stimme zu erheben und ihren Protest gegen Missstände der heutigen Zeit zu äußern in den Fußstapfen Martin Luthers, der rund 500 Jahre zuvor mit seinen 95 Thesen gegen den Ablasshandel und weitere Missstände innerhalb der Kirche protestiert und damit den 
Anstoß zu einer Kirchenreformbewegung gegeben hatte. Mit dem Aufruf waren die Fragen verbunden, wogegen in der heutigen Welt protestiert werden müsse, was im Bereich von Kirche und Schule zu erneuern sei und wie eine gute Welt, eine gute Kirche und eine gute Schule aussehen sollen.

Insgesamt beteiligten sich Schülerinnen und Schüler verschiedener Altersgruppen von über 60 Schulen aus vier Kontinenten an der Aktion. Die Beiträge erfolgten mehrheitlich in Form von Sammlungen einzelner plakativer Statements, die im Rahmen der Aktion als "Thesen “ bezeichnet wurden. ${ }^{2}$ Insgesamt gingen 1167 solcher Thesen ein. Tabelle 1 zeigt die Verteilung der Beiträge auf Länder und Kontinente.

\begin{tabular}{|l|c|c|}
\hline Land & $\begin{array}{c}\text { Anzahl der } \\
\text { beteiligten } \\
\text { Schulen }\end{array}$ & $\begin{array}{c}\text { Anzahl der } \\
\text { Thesen }\end{array}$ \\
\hline Afrika & 31 & 697 \\
\hline Dem. Republik Kongo & 14 & 265 \\
\hline Kamerun & 11 & 339 \\
\hline Ruanda & 4 & 45 \\
\hline Ghana & 2 & 30 \\
\hline Südamerika & 19 & 44 \\
\hline Argentinien & 18 & 3 \\
\hline Brasilien & 9 & 41 \\
\hline Europa & 6 & 315 \\
\hline Deutschland & 2 & 291 \\
\hline Slowakei & 1 & 16 \\
\hline Schweiz & 1 & 8 \\
\hline Nordamerika & 1 & 32 \\
\hline USA & ohne Angabe & 32 \\
\hline Asien & ohne Angabe & 97 \\
\hline Hong Kong 60 & 1167 \\
\hline Philippinen & & 53 \\
\hline Summe & Angabe & 44 \\
\hline
\end{tabular}

Tab. 1: Anzahl der beteiligten Schulen und der eingesandten Statements nach Kontinenten und Ländern,

Quelle: eigene Darstellung

In der Auswertung der Statements wurden 60 verschiedene Themen identifiziert, die den Kategorien „Welt“ bzw. „Gesellschaft", „Kirche“ und „Schule“ zugeordnet wurden. ${ }^{4}$ Aussagen zu den Sachbereichen, die hier besonders betrachtet werden (Globalisierung, Bildung sowie religiöse Aspekte wie Bibel und Glauben), wurden besonders markiert und - nach den identifizierten Themen gruppiert - inhaltsanalytisch ausgewertet.

Die Thesen der Schülerinnen und Schüler wurden in Deutsch, Englisch und Französisch eingesandt und seitens GPENreformation in alle diese Sprachen übersetzt. Zur besseren Lesbarkeit wird hier jeweils die deutsche Version angegeben; auch die Daten wurden in der jeweiligen deutschsprachigen Version ausgewertet.

\section{Kritik und Visionen zu Weltgesellschaft, Schule und Kirche}

Ein erster auswertender Überblick über eingesandte Statements ${ }^{5}$ hat gezeigt, dass die meisten Schülerinnen und Schüler ihren Alltag und ihre Umwelt recht wach und kritisch wahrnehmen: sie zeigen eine hohe Sensibilität für Recht und Unrecht, für Anstand und Fehlverhalten, sie formulieren mutige Aussagen zur Verwerflichkeit von Korruption und gewaltsamen Konflikten und fordern zum dringenden Kampf gegen Armut und Umweltzerstörungen auf.

In den Thesen, die eine Vision einer besseren Weltgesellschaft zum Ausdruck bringen, betonen die Schülerinnen und Schüler an erster Stelle den Protest gegen Ungerechtigkeit. Die Jugendlichen fordern eine gerechtere Justiz, die Gleichbehandlung aller Menschen und eine gerechtere Verteilung von Gütern. Damit verbunden ist der Wunsch nach friedvollem Zusammenleben, besserer materieller Versorgung, besseren demokratischen Strukturen und einer allgemeinen Anerkennung und Einhaltung der Menschenrechte. Einem guten menschlichen Miteinander messen die Jugendlichen einen hohen Stellenwert für die Zukunft bei, wenn formuliert wird: „Wir wünschen uns, dass die Menschen in unserem Land grundsätzlich netter und positiver gegenüber anderen werden." $(834)^{6}$ Während aus den afrikanischen Ländern oft ein vehementer Protest gegen Korruption formuliert und die Forderung nach Gleichbehandlung und Transparenz im Umgang mit Geld gefordert wird, ${ }^{7}$ ist Jugendlichen aus Europa und aus Südamerika vor allem an Umweltschutz und Bewahrung der natürlichen Ressourcen gelegen.

Im Hinblick auf die Schule richtet sich die Kritik der Jugendlichen auf ein mangelhaftes Schulklima und einen zu wenig anerkennenden Umgang zwischen Lehrenden und Lernenden. Das Ausschließen Schwächerer, Prügeleien, Erpressungen auf dem Schulhof und Mobbing werden beanstandet, wie auch ungerechte Behandlung durch Lehrkräfte. „Viele Lehrkräfte respektieren die Rechte der Kinder nicht. Sie bestrafen die Kinder ohne guten Grund." (123) Die Zugänglichkeit von Bildung für alle sowie eine bessere Qualifizierung und Bezahlung der Lehrkräfte sind ein besonders in Afrika oft vorgetragenes Problem. Eine gute Schule sollte aus Sicht der Jugendlichen gute Rahmenbedingungen für das Lernen schaffen.

Auch im Hinblick auf die Kirche wird das Verhalten einzelner Personen, insbesondere leitender Persönlichkeiten, kritisiert. Der Umgang mit Geld oder die Ausnutzung von Positionen zum eigenen Vorteil werden vor allem in Afrika als ein Problem formuliert. Gutes Verhalten ist eine christliche Tugend; denn Christen „sollen das Evangelium predigen, und Jesus als der Inhalt der Botschaft wird ihnen sagen, was sie tun sollen“ (665). In Europa liegt ein Gewicht auf dem Wunsch nach erneuerten Kirchenstrukturen und einer zeitgemäßeren Form des Glaubens. Auch wird die Uneinigkeit der Kirchen und Religionen kritisiert; „schließlich ist es doch derselbe Gott, an den wir glauben" (1165). Daneben protestieren die Jugendlichen gegen die gegenseitige Abschottung der (Welt-)Religionen voneinander und fordern mehr Offenheit und gegenseitige Akzeptanz.

Die eingesandten Thesen weisen in vielen Punkten große Gemeinsamkeiten auf, gerade wenn es um grundlegende Visionen oder Werte geht. Es finden sich aber natürlich auch 
Unterschiede aufgrund der unterschiedlichen Lebensbedingungen in den verschiedenen Ländern. Die eingesandten Statements aus Europa, Afrika und Asien lassen Unterschiede beispielsweise hinsichtlich der Bedeutung von Frömmigkeit und Religion oder unterschiedlicher Erfahrungen der christlichen Jugendlichen als religiöse Mehrheit oder Minderheit in ihren jeweiligen Gesellschaften erkennen. Religion, Traditionen und kirchliches Leben haben vor allem für die Teilnehmenden aus afrikanischen Ländern einen hohen Stellenwert. Dort wie auch in Teilen Asiens sind Korruption, soziale Spannungen und Armut ein brennendes Problem. Unterschiede zwischen den Teilnehmenden verschiedener Weltregionen finden sich auch bei deren Positionen zu Folgen von Globalisierung, zur Rolle von kulturellen und religiösen Traditionen und zu sexuellen Normen. ${ }^{8}$

Nach dieser einführenden Übersicht soll im nächsten Abschnitt der Frage nachgegangen werden, welches Bild von Globalität sich in den Statements findet. Zudem soll analysiert werden, welche Aussagen zu Bildung gemacht werden, da in auffällig vielen Einsendungen eine bessere Bildung explizit als die Schlüsselqualifikation angesehen wird, mit der eine bessere Zukunft aufgebaut werden kann. Zahlreiche Texte begründen ihre Aussagen mit dem Bezug auf die christliche Lehre oder speziell auf die Bibel. Dies soll ebenfalls im Folgenden genauer dargestellt werden.

\section{Globales Bewusstsein, Bildung und Religion - interpretierende Auswertung Aspekte der Globalisierung}

In einem Großteil der Statements finden sich Formulierungen, die eine globale Perspektive vermuten lassen, ohne dass diese jedoch explizit benannt wird. Dies ist beispielsweise der Fall, wenn von einer „besseren Welt" gesprochen wird. Um jedoch herauszuarbeiten, welche Perspektiven die Jugendlichen konkret auf Globalität bzw. Globalisierung und ihre Folgen haben, wurden die Statements auf explizite Aussagen zu diesem Thema durchgesehen. Dabei zeigte sich, dass die Jugendlichen Globalität vor allem im Kontext von internationalen Beziehungen, wirtschaftlichen Entwicklungen und Abhängigkeiten, sozialen Netzwerken und im Zusammenhang mit Migration und Flucht wahrnehmen.

Die das Thema Globalität ansprechenden Statements nehmen dabei unterschiedliche Perspektiven ein. Häufig wird Bezug auf lokale Räume jenseits des eigenen Nahraums genommen, indem Missstände in anderen Ländern angesprochen werden. Ein anderer Blickwinkel findet sich dort, wo Menschen verschiedenen Gemeinschaften zugeordnet werden, die dann - je nach Thema - als die eigene oder eine fremde Gemeinschaft dargestellt werden. Zudem kommt Globalität darin zum Ausdruck, dass Strukturaspekte oder übergreifende Phänomene benannt werden, wie in der Aussage: „Eine gute Gesellschaft lässt nicht zu, dass das Konsumverhalten der Reichen die ganze Welt zerstört." (1218)

Auffällig ist, dass sich die Schülerinnen und Schüler gegenüber Globalisierung mehrheitlich kritisch äußern, wenn sie diese explizit ansprechen oder auf deren Folgen hinweisen. Aber es werden auch positive Aspekte der Globalisierung genannt, wenn allgemein auf „Organisationen [...], die sich für
Frieden einsetzen" (1197) hingewiesen wird oder ganz konkret die Vereinten Nationen (910) genannt werden. Im Alltagsleben empfinden die Jugendlichen soziale Netzwerke mittels digitaler Medien einerseits als nützlich, um „untereinander zu kommunizieren“ (267) und „ihre gemeinsamen Interessen mit anderen Weltregionen [auszutauschen] “ (653), andererseits jedoch auch als problematisch, da aufgrund der darin wahrgenommenen allgemeinen Orientierung an westlichen Standards „die traditionellen Werte der Afrikaner verloren [gehen]" (653). Weiterhin benennen die Jugendlichen als negative Folgen von Globalisierung konkret die wirtschaftliche Abhängigkeit der Länder des globalen Südens von Industrieländern, kulturelle, religiöse und ethnische Konflikte, Terrorismus, Ausländerfeindlichkeit und den Verlust von sozialen Werten wie z.B. den Bedeutungsverlust der Familie als sozialer Bezugsgruppe.

Die Mehrheit der Statements, die Globalität oder Globalisierung ansprechen, sind jedoch als Forderungen formuliert, wie eine globale Welt gestaltet sein soll. Wie bereits erwähnt, lässt sich diese Perspektive häufig nur erahnen; etwa, wenn generell Frieden oder Gerechtigkeit gefordert werden. Konkrete Erwartungen richten sich unter anderem auf die konsequente Einführung demokratischer Staatsformen in allen Ländern und den Ausgleich von wirtschaftlichen Ungleichverteilungen. Es wird als ungerecht empfunden, „dass das wirtschaftliche Wachstum, welches eine Folge der Globalisierung ist, nicht nur dem einen Prozent der Weltbevölkerung Vorteile verschafft, das 50\% des Gesamtvermögens der Erde besitzt" (1122). Ebenso wird die Aufnahme von Kriegsflüchtlingen durch alle Länder und insbesondere menschlicher Umgang, faire Behandlung, Rücksicht und Schutz, zumindest aber Toleranz gegenüber Flüchtlingen gefordert.

Vor dem Hintergrund, dass die Thesen im Kontext des evangelischen Schulwesens entstanden sind, überrascht es nicht, dass die Jugendlichen auch von ihren Kirchen eine konkrete Beteiligung an der positiven Gestaltung einer globalisierten Welt erwarten; beispielsweise sollen sich die Kirchen „in die Aktivitäten der Regierung zur Erhaltung des weltweiten Friedens einbringen“ (218) oder „dafür einsetzen, dass sich die Schere zwischen Arm und Reich nicht weiter öffnet" (1122).

\section{Bildung und Bildungsgerechtigkeit}

Im Bereich der Bildung nehmen die Jugendlichen verschiedene Missstände und Unzulänglichkeiten wahr, die sich vor allem auf den gleichen Zugang zu Bildung für alle beziehen. Vor allem Mädchen, sozial schwächer Gestellte, Flüchtlinge und Menschen mit Behinderungen werden als benachteiligt wahrgenommen, womit die Forderung nach Gleichheit aller einhergeht - zum Teil religiös begründet in der Annahme, dass alle Menschen gleichermaßen „nach dem Bilde Gottes geschaffen sind“ (531). Auch innerhalb der Kirche wird diese Ungleichbehandlung von Mädchen und Jungen in Bezug auf Bildung wahrgenommen. Weiterhin wird vehemente Kritik an Korruption innerhalb des Bildungswesens geübt, da diese soziale Ungleichheit begünstige. Darüber hinaus verurteilen Jugendliche dies als moralisch verwerflich.

Daneben fordern die Jugendlichen Bildungsqualität sowohl hinsichtlich schulischer Angebote als auch im außerschulischen und kulturellen Bereich. Bildung als „Grundlage 
jeder Gesellschaft“ (260) soll auf Demokratiefähigkeit abzielen und somit zur Beseitigung von Konflikten beitragen. Eine höhere Qualität im Bereich der beruflichen Bildung kann nach Ansicht der Jugendlichen darüber hinaus zur Behebung weiterer gesellschaftlicher Probleme beitragen. Eine Schlüsselfunktion nimmt dabei in der Wahrnehmung der Jugendlichen auch die Lehrerbildung ein.

Auf staatlicher bzw. politischer Ebene fordern die Jugendlichen, dass Bildung eine gewisse Priorität vor anderen Themen eingeräumt werden solle; in diesem Zusammenhang protestieren die Jugendlichen zum einen gegen die schlechte materielle und finanzielle Ausstattung einiger Schulen, zum anderen weisen sie auf die Reformbedürftigkeit von Bildungssystemen und -plänen in einigen Ländern hin.

Mit (zum Teil konkret benanntem) Bezug auf das Programm „Education for All“ der Vereinten Nationen erfolgt seitens einzelner junger Erwachsener die Forderung nach konkreteren Maßnahmen auf nationaler Ebene, die zur Erreichung dieses Ziels unabdingbar sind. Hierfür wird internationale Kooperation gefordert sowie die Koordination verschiedener Akteure, deren Zusammenarbeit bisher als wenig aufeinander abgestimmt wahrgenommen wird.

\section{Religion und Glauben als Bezugspunkte}

In zahlreichen Statements zu allen Bereichen („Kirche“, „Schule“ und „Welt") finden sich explizit religiöse Bezüge zu christlichen Glaubensüberzeugungen, teilweise wird direkt auf die Bibel in ihrer Gesamtheit oder auf konkreten Bibelstellen Bezug genommen.

Die Jugendlichen sehen in der Bibel, in Gottes Wort oder speziell in der Lehre und den Weisungen von Jesus Christus grundlegende Orientierungen. In einer gefühllosen Gesellschaft könne die biblische Botschaft Hilfe anbieten, zu Gelassenheit und einer friedvollen und gütigen Einstellung führen (1304), Regeln für ein friedlicheres Leben aufzeigen (1249) und zu einer inneren Freiheit von den materiellen Zwängen des Alltagslebens verhelfen (1255). Gott, dem man die Sorge für die Zukunft überlassen könne (1254), verspreche Segnungen und Frieden des Herzens (196 und 848), was zu einer besseren Zukunft in Respekt voreinander führe (1250). Gott, der einen Plan habe, würde dafür Sorge tragen, dass alles gut werde (1251) und dass man in allem einen Sinn erkennen könne (1252). In einem Statement wird Gott um Beistand gebeten, um anderen helfen und um sicher von der Arbeit nach Hause kommen zu können (573). In einem Votum heißt es sogar, der christliche Glaube würde helfen, sich im Alltag besser zu konzentrieren, und ein Besuch der Kirche könne Stress lindern und die Welt friedlicher machen (1306). Eine besondere didaktische Rolle spielt die Bibel im folgenden Statement: „Ich protestiere gegen Analphabetismus; denn die Bibel ist ein Licht auf unserem Pfad (Psalm 119, 105).“ (1329) Jedoch findet sich auch die Bemerkung, dass manche Menschen die Bibel so interpretieren, wie es ihnen passe (606).

Das Stichwort Nächstenliebe spielt in einer Reihe von Thesen eine zentrale Rolle: die christliche Botschaft sei als Verkündigung von Liebe zu verstehen (1191), der Glaube an Gott leite zu Liebe und Engagement für andere an (532) sowie dazu, Schwachen und Entrechteten beizustehen $(1191,1288)$. In der
Bibel fänden sich zahlreiche gute Beispiele zur Vermeidung von Fehlverhalten (3) und gegen die Ausbeutung von Menschen (1251); einige Bibelstellen gegen Diebstahl und Gewalt (847; 1199) werden angeführt, der Hinweis auf das Tötungsverbot wird auf extremistische muslimische „Attentäter mit Bombe“ bezogen (637). Ein Protest gegen Hunger und Flucht wird verbunden mit einem Hinweis auf Abraham und Isaak als „Wirtschaftsflüchtlinge“ (1207).

Ein weiteres mehrfach genanntes Thema ist die theologische Begründung der Gleichheit aller Menschen. Nach der Überzeugung der Schüler einer Schule sei diese darin begründet, dass die Menschen nach Gottes Bild geschaffen seien (531). Auch die Lehre Jesu würde die Gleichheit der Menschen vor Gott und die Respektierung der Würde des Menschen betonen $(1250 ; 1388)$. Daraus ergebe sich auch der Rechtsschutz für Fremde (1209). Für Vorbehalte gegenüber Menschen anderer Herkunft oder Hautfarbe fänden sich jedoch auch Geschichten in der Bibel wie beispielsweise die Berichte über das Ergehen des Volkes Israel in Ägypten (1289). Aufgrund des christliches Verständnisses von Würde und Ebenbildlichkeit Gottes müsse man gegen Folter und Grausamkeiten aufstehen (1191). Mit der Überzeugung, dass Gott diese Welt geschaffen habe, wird mehrfach eine besondere Aufmerksamkeit und ein entschlossenes Eintreten für die Bewahrung der Erde als natürlichem Lebensraum und den Schutz der Artenvielfalt begrün$\operatorname{det}(472 ; 1216 ; 1217)$. Auch religiöse Vorbilder spielen eine wichtige Rolle. Jesus stellt für die Jugendlichen ein solches Vorbild dar (1191), aber auch Martin Luther, der Missstände angeprangert habe und dessen Werk weitergeführt werden müsse (851). Der Papst sei mit seiner „grünen Enzyklika“ ein Verfechter des Schutzes und Erhalts der natürlichen Lebensgrundlagen (1219).

Spezielle Erwartungen richten sich an die Kirche, die christliche Überzeugungen wirksam vertreten solle. So soll die Kirche den Gemeinschaftsgeist aller Menschen stärken und selbst Zeichen des Friedens und der Einheit sein (1233). Sie sollte Menschenrechte unterstützen (1193), Flüchtlingen helfen $(1202 ; 1211)$ und einen fairen Handel fördern, da dieser einen Beitrag zu nachhaltiger Entwicklung fördere (1227). Andererseits wird vereinzelt die Gleichstellung von Frauen in kirchlichen Ämtern mit Bezug auf konkrete Bibelstellen abgelehnt, wie das folgende Statement aus Afrika zeigt: „Wir prangern an, dass Frauen als Pfarrerinnen geweiht werden und ihnen die Verantwortlichkeit für eine Kirche übertragen wird mit dem Ziel, das Eintreiben von Spenden zu vereinfachen (Tim 2, 12; Tim 3,1-2; Kor 14, 34).“ (403)

Ein besonderer Themenbereich, der mit biblischen Positionen in Verbindung gebracht wird, ist die Sexualität. Ehebruch und unüberlegter Sex seien Sünde und stünden im Widerspruch zur Lehre der Bibel (595). Mehrere ablehnende Bewertungen erhält Homosexualität, die mit dem Bezug auf den alttestamentlichen Bericht über Sodom und Gomorrha (Genesis 19, 4-5) bzw. mit Hinweis auf die Bibelstelle Lukas 17, 30 als ein Laster bzw. als Sünde angesehen wird (373; 379); jedoch stammen diese Aussagen ausschließlich von Schülerinnen und Schülern aus Kamerun und der Demokratischen Republik Kongo. Vereinzelt entnehmen Jugendliche der Bibel, dass afrikanische Politiker nicht durch Weiße gezwungen werden dürften, Homosexualität gutzuheißen (607). Ebenso wer- 
den protestantische Kirchen kritisiert, die Homosexualität gutheißen, „obwohl sie eine Sünde ist“ (374). Menschen mit anderen Überzeugungen müssen in ihrem religiösen Glauben respektiert werden (1306). Es gebe jedoch auch Menschen, die nicht „an spirituelle Texte glauben“ (1263) oder deren Wahrheit missachten würden (1254). Die Vielfalt der Religionen sei ein Hindernis für die Einheit (1249). Bei der Aufnahme von Flüchtlingen dürfte aber deren Religion keine Rolle spielen (1213).

Man kann bilanzieren, dass für eine Reihe von Schülerinnen und Schülern eine christliche und biblische Fundierung ihrer Überzeugungen wichtig ist und sie diese auch darlegen und begründen können. Neben einem grundsätzlichen Vertrauen, das sich daraus speist, sind Werte wie Achtung und die Fürsorge für andere, die Gleichheit der Menschen und die Sorge um den Erhalt der natürlichen Umwelt sehr wichtig, ebenso wie die Vorbildfunktion einiger christlicher Persönlichkeiten.

\section{Religiöse Bildung in einer globalisierten Welt - Zusammenfassung der Befunde}

Auf der Basis der dargestellten Befunde sollen zusammenfassend einige Antworten auf die eingangs gestellten Fragen gegeben werden. In zahlreichen Statements wird sichtbar, dass Jugendliche einen globalen Bezugshorizont wahrnehmen und in verschiedenen Aspekten Zusammenhänge zwischen ihrem Alltag und weltweiten Entwicklungen sehen. Einerseits finden sich die sehr umfassenden Visionen einer gerechteren und friedvollen Welt, andererseits aber auch spezifische Strukturen internationale Zusammenhänge, vor allem im Hinblick auf die Verteilung von Reichtum und Armut, wirtschaftliche Gerechtigkeit, Umweltzerstörung und Migration. Die Jugendlichen wissen auch von internationalen Organisationen und Netzwerken, die in diesem Feld tätig sind. Die weltweiten Kommunikationsmöglichkeiten werden als Chance gesehen, Gefährdungen der Globalisierung vor allem im Bereich der Familie und traditioneller Sozialstrukturen.

Was bedeutet Schülerinnen und Schülern Bildung und Lernen in diesem Kontext? Die eingereichten Statements lassen erkennen, dass Bildung und Lernen als unbestrittener Wert angesehen und geschätzt werden. Der Wunsch nach besserer personeller und finanzieller Ausstattung von Schulen, nach verbesserter Unterrichtsqualität, nach einem Zugang aller zu angemessener Bildung zeigt die Bedeutung an, die Schülerinnen und Schüler in den Bildungsangeboten und den Chancen sehen, die ihnen damit für ihre persönliche Zukunft eröffnet werden.

Welche Werte sind ihnen wichtig und welche $\mathrm{Zu}$ kunftsvisionen haben sie? Welche Rolle spielen religiöse Überzeugungen dabei? Auf diese Fragen kann man antworten, dass die Thesen der jungen Leute vielfach einen Bezug zu einem umfassenden Gemeinwohl aller erkennen lassen. Rücksichtnahme und Fürsorge für andere, besonders für Minderheiten und Schwächere, die Gleichheit aller Menschen, verantwortlicher Umgang mit der Natur, ein demokratisches Gemeinwesen, Regeln für ein faires Miteinander und ein friedvolles Leben in innerer Freiheit sind durchgehend zu findende Werte und explizierte Überzeugungen, die sowohl eine allgemein humane Dimension, aber auch eine religiöse Grundlage haben. Egois- tische und rechthaberische Positionen sind selten zu finden. Bei einem Teil der Schülerinnen und Schüler ist der Bezug auf den christlichen Glauben und die Bibel als deren grundlegende Schrift explizit ausgeführt. Sicherlich ist die in einigen Statements erkennbare gute Bibelkenntnis im Unterricht und der Ausrichtung evangelischen Schulen begründet. Jedoch werden ähnliche Werte und Überzeugungen auch dort geteilt, wo diese nicht explizit auf christliche Quellen bezogen werden.

\section{Sensibilisierung junger Menschen für globale Herausforderungen - pädagogische Perspektiven}

Drei Folgerungen können aus dieser Analyse gezogen, hier aber nur angedeutet werden:

- Zahlreiche Jugendliche haben im Rahmen der Aktion „Protest für die Zukunft“ die Möglichkeit wahrgenommen, sich zu umfassenden Fragen ihrer Zukunft zu äußern. Solche Möglichkeiten der Artikulation sollten so oft wie möglich angeboten werden, da damit ein Nachdenken über derartige Fragen als auch ein damit verbundenes Verantwortungsbewusstsein gefördert werden. Die eingesandten Thesen lassen erkennen, dass zahlreiche Statements von Schülergruppen oder sogar einer ganzen Schule erarbeitet worden sind. Das bietet die Möglichkeit zu internen Diskussionsprozessen. Der von dem weltweiten Netzwerk evangelischer Schulen angeregte Austausch zwischen Schulen über Länder- und oder kontinentale Grenzen hinweg kann den Horizont der Jugendlichen deutlich erweitern. Damit ist der zweiten Punkt bereits angesprochen.

- Weltweite Vernetzungen, wie die christlichen Kirchen aber auch Schulpartnerschaften sie anbieten, sind eine große Chance, Schülerinnen und Schüler mit globalen Zusammenhängen vertraut zu machen und ihnen Werte zu vermitteln, die eine gemeinsame Verantwortung für Frieden und Gerechtigkeit fördern. Sie können sich selbst als einen aktiven Teil eines globalen Netzwerkes verstehen lernen. Die Verbindung solcher Vernetzungen mit Sprach-, Geschichts-, Geographie- oder Religions- bzw. Ethikunterricht sowie mit Schüleraustausch bieten mehrdimensionale Lernmöglichkeiten.

- Wie die oben erwähnten Beispiele zeigen, bilden biblische Geschichten und Gebote Haftpunkte, mit denen aktuelle alltägliche Zusammenhänge, denen Schülerinnen und Schüler begegnen, gedeutet und zugeordnet werden können. Diese helfen den Jugendlichen, eine Brücke herzustellen zwischen ihren religiösen Überzeugungen, ihrem alläglichen Nahraum, geschichtlichen und politischen Zusammenhängen und globalen Herausforderungen, die oftmals nur in großen abstrakten Kategorien beschrieben werden können. (Religions-)Pädagogische Maßnahmen sollten Schülerinnen und Schüler daher in einem reflektierten Umgang mit orientierenden Texten, Geschichten und Persönlichkeiten bestärken und diesen fördern. 
Pädagogisches Handeln steht vor der Herausforderung, junge Menschen dazu zu befähigen, die Welt aktiv mitzugestalten. Jugendlichen, die oft ein ausgeprägtes Gespür für Veränderungsbedarf in der Welt haben, in der sie leben, sollten Spielräume und Lernfelder eröffnet werden, um selbst zu der Veränderung beitragen zu können. Religiösen Ansichten muss dabei wertschätzend begegnet werden - in vielen Fällen stellen die religiösen Ansichten der Jugendlichen eine nicht zu unterschätzende Motivation dazu dar, für Veränderungen einzustehen. Dies zu fördern und gleichzeitig religiösen Dialog und Offenheit für andere Ansichten anzuregen, bleibt eine der zentralen Aufgaben von Pädagogik in einer globalisierten Welt.

\section{Anmerkungen}

1 Nähere Informationen zu diesem Netzwerk siehe https://www.gpenreformation. net; siehe auch den Sammelband von Simojoki, Scheunpflug \& Schreiner (2018).

2 Ausgewiesenes Ziel der Aktion war unter anderem die Zusammenstellung von 95 Thesen von Schülerinnen und Schülern evangelischer Schulen weltweit", die unter https://www.gpenreformation.net/networkactivities/projekte-fur-schulergruppen/protest-for-the-future/ in drei Sprachen veröffentlicht wurden.

3 Die genaue Anzahl der beteiligten Schulen ist nicht feststellbar, da sich die Schulen aus Hong Kong und den Philippinen aus Datenschutzgründen mit einer gemeinsamen Einsendung an der Aktion beteiligten, in der lediglich Angaben zu den Altersgruppen der Schülerinnen und Schüler gemacht wurden.

4 Die Aufbereitung und Auswertung der Daten erfolgte mithilfe der Software MAXQDA. Bei der Aufbereitung und Codierung der Thesen sowie deren inhaltlicher und statistischer Auswertung wurden die Autorin und der Autor von Lea Wiebke Markus und Kilian Schmidt, Otto-Friedrich-Universität Bamberg, unterstützt. Beiden gebührt hierfür ein herzlicher Dank.

5 Eine 2016 vorgelegte Auswertung (Affolderbach \& Plötz, 2016) bezog sich auf Einsendungen vor Abschluss der Einsendefrist. In diesem Abschnitt werden wesentliche Schwerpunkte dieser Auswertung zusammenfassend wiedergegeben.

6 Die Zahlenangaben beziehen sich auf die Zeilen der zur Datenerfassung erfolgten Zusammenstellung der Statements.
7 Zur Nähe mancher Konstellationen in afrikanischen Ländern zur Situation während der Reformationszeit vgl. Affolderbach, 2018.

8 Ein umfassender Vergleich der Thesen von Jugendlichen verschiedener Kontinente und/oder Länder kann an dieser Stelle aus Platzgründen nicht erfolgen.

\section{Literatur}

Affolderbach, M. \& Plötz, E. (2016). Protest für die Zukunft - Kritik und Visionen von Schülerinnen und Schülern aus Evangelischen Schulen weltweit, epd-Dokumentation, 35(16), 35-42.

Affolderbach, M. (2018). Luther in Afrika. Anmerkungen zur Aktualität der Reformation. In H. Simojoki, A. Scheunpflug \& M. Schreiner (Hrsg.), Evangelische Schulen und religiöse Bildung in der Weltgesellschaft. Die Bamberger BarbaraSchadeberg-Vorlesungen (S. 191-202). Münster: Waxmann.

Simojoki, H., Scheunpflug, A. \& Schreiner, M. (Hrsg.) (2018). Evangelische Schulen und religiöse Bildung in der Weltgesellschaft. Die Bamberger Barbara-SchadebergVorlesungen. Münster: Waxmann.

\section{Dr. Martin Affolderbach}

war bis 2012 Oberkirchenrat / Referent für Islam und Weltreligionen im Kirchenamt der Evangelischen Kirche in Deutschland und nimmt Lehraufträge unter anderem in Bamberg und Nürnberg wahr.

\section{Evi Plötz}

ist wissenschaftliche Mitarbeiterin und Doktorandin am Lehrstuhl für Allgemeine Pädagogik an der Otto-Friedrich-Universität Bamberg. 